\title{
Scaling Symmetry and Integrable Spherical Hydrostatics
}

\author{
Sidney Bludman ${ }^{1}$, Dallas C. Kennedy ${ }^{2}$ \\ ${ }^{1}$ Departamento de Astronomía, Universidad de Chile, Santiago, Chile \\ ${ }^{2}$ Natick, USA \\ Email: sbludman@das.uchile.cl,dalet@stanfordalumni.org
}

Received October 17, 2012; revised December 10, 2012; accepted December 25, 2012

Copyright (C) 2013 Sidney Bludman, Dallas C. Kennedy. This is an open access article distributed under the Creative Commons Attribution License, which permits unrestricted use, distribution, and reproduction in any medium, provided the original work is properly cited.

\begin{abstract}
Any symmetry reduces a second-order differential equation to a first integral: variational symmetries of the action (exemplified by central field dynamics) lead to conservation laws, but symmetries of only the equations of motion (exemplified by scale-invariant hydrostatics) yield first-order non-conservation laws between invariants. We obtain these nonconservation laws by extending Noether's Theorem to non-variational symmetries and present an innovative variational formulation of spherical adiabatic hydrostatics. For the scale-invariant case, this novel synthesis of group theory, hydrostatics, and astrophysics allows us to recover all the known properties of polytropes and define a core radius, inside which polytropes of index $n$ share a common core mass density structure, and outside of which their envelopes differ. The Emden solutions (regular solutions of the Lane-Emden equation) are obtained, along with useful approximations. An appendix discusses the $n=3$ polytrope in order to emphasize how the same mechanical structure allows different thermal structures in relativistic degenerate white dwarfs and zero age main sequence stars.
\end{abstract}

Keywords: Lagrangian Mechanics; Symmetry; Hydrodynamics; Astrophysics; Stellar Structure

\section{Symmetries of Differential Equations and Reduction of Order}

Noether's Theorem relates every variational symmetry, a symmetry of an action or similar integral, to a conservation law, a first integral of the equations of motion [1]. By an extension of Noether's Theorem, non-variational symmetries - symmetries of the equations of motion which are not in general variational symmetries - also lead to first integrals, which are not conservation laws of the usual divergence form, as discussed in a previous article [2]. There it was shown that a Lagrangian $\mathcal{L}\left(t, q_{i}, \dot{q}_{i}\right)$ and action $S=\int \mathcal{L}\left(t, q_{i}, \dot{q}_{i}\right) \mathrm{d} t$, with degrees of freedom $q_{i}$, can be transformed under an infinitesimal point transformation $\delta\left(t, q_{i}\right), \delta q_{j}\left(t, q_{i}\right)$ :

$$
\begin{aligned}
\delta \mathcal{L} & =\dot{\mathcal{L}} \delta t+\left(\partial \mathcal{L} / \partial q_{i}\right) \delta q_{i}+\left(\partial \mathcal{L} / \partial \dot{q}_{i}\right)\left[\frac{\mathrm{d} \delta q_{i}}{\mathrm{~d} t}-\dot{q}_{i} \frac{\mathrm{d} \delta t}{\mathrm{~d} t}\right] \\
& =\left[\frac{\mathrm{d} G}{\mathrm{~d} t}-\mathcal{L} \cdot \frac{\mathrm{d}(\delta t)}{\mathrm{d} t}+\mathcal{D}_{i} \cdot\left(\delta q_{i}-\dot{q}_{i} \delta t\right)\right],
\end{aligned}
$$

in terms of the total derivative of the Noether charge, $G:=\mathcal{L} \cdot \delta t+p_{i} \cdot\left(\delta q_{i}-\dot{q}_{i} \delta t\right)$, and the variational derivative $\mathcal{D}_{i}:=\partial \mathcal{L} / \partial q_{i}-\mathrm{d}\left(\partial \mathcal{L} / \partial \dot{q}_{i}\right) / \mathrm{d} t$. For transformations that leave initial and final states unchanged, the variation in action is

$$
\delta S_{i f}=G(f)-G(i)+\int_{i}^{f} \mathrm{~d} t\left[\delta q_{i} \cdot \mathcal{D}_{i}+\delta t \cdot\left(\frac{\mathrm{d} \mathcal{H}}{\mathrm{d} t}+\frac{\partial \mathcal{L}}{\partial t}\right)\right],
$$

if the term in $\mathrm{d}(\delta t) / \mathrm{d} t$ is integrated by parts. If the system evolution obeys an action principle, that this variation vanish for independent variations $\delta q_{i}, \delta t$ that vanish at initial and final times, the system obeys the Euler-Lagrange equations $\mathcal{D}_{i}=0$ and $\mathrm{d} \mathcal{H} / \mathrm{d} t=-\partial \mathcal{L} / \partial t$, the rate of change of the Hamiltonian in non-conservative systems. On-shell, where $\mathcal{D}_{i}=0$,

$$
\begin{aligned}
& \delta S_{i f}=\int_{i}^{f} \bar{\delta} \mathcal{L} \mathrm{d} t=G(f)-G(i) \\
& \frac{\mathrm{d} G}{\mathrm{~d} t}=\bar{\delta} \mathcal{L}:=\delta \mathcal{L}+\mathcal{L} \cdot(\mathrm{d} \delta t / \mathrm{d} t) .
\end{aligned}
$$

This is Noether's equation, giving the evolution of a symmetry generator or Noether charge, in terms of the Lagrangian transformation that it generates. It expresses the Euler-Lagrange equations of motion as the divergence of the Noether charge. This divergence vanishes 
for a variational symmetry, but not for any other symmetry transformation.

Noether's Equation (4) could have been derived directly from the definition of the Noether charge. But using the action principle makes manifest the connection between Noether's equation and the Euler-Lagrange equations. We use the action principle and this connection to reformulate the theory of hydrostatic barotropic spheres, which is integrable if they are scale symmetric, even where this scale symmetry is not a symmetry of the action (Section 2). The first integrals implied by any symmetry of the equations of motion, while generally not vanishing-divergence conservation laws, are still useful dynamical or structural first-order relationships.

Because it neglects all other structural features, scaling symmetry is the most general simplification that one can make for any dynamical system. For the radial scaling transformations we consider, $\delta r=r$, the Lagrangian scales as some scalar density $\delta \mathcal{L}=-2 \tilde{\omega} \mathcal{L}$; and the action scales as $\delta S=(1-2 \tilde{\omega}) S$. The Noether charge generating the scale transformation evolves according to a non-conservation law $\mathrm{d} G / \mathrm{d} t=(1-2 \tilde{\omega}) \mathcal{L}$, a first-order equation encapsulating all of the consequences of scaling symmetry [2]. From this first-order equation follow directly all the properties of index- $n$ polytropes, as established in classical works $[3,4]$, modern textbooks $[5,6]$, and the recent, excellent treatments of Horedt and Liu $[7,8]$.

Our secondary purpose is to present an original variational formulation of spherical hydrostatics and to extend Noether's Theorem to non-variational scaling symmetry, which yields a scaling non-conservation law (Section 2). For spherical hydrostasis, we define a core radius, inside which all stars exhibit a common mass density structure. Outside this core, polytropes of different index $n$ show different density structures as the outer boundary is felt (Section 3). Section 4 completes the integration of the Lane-Emden equation by quadratures and obtains useful approximations to the Emden function $\theta_{n}(\xi)$.

An appendix reviews the thermodynamic properties of the physically important polytropes of index $n=3[2,5,6]$. What is original here is the explanation of the the differences between relativistic degenerate white dwarf stars and ideal gas stars on the zero-age main sequence (ZAMS), following from their different entropy structures. Our original approximations to $\theta_{3}(\xi)$ should prove useful in such stars.

\section{Scaling Symmetry and Integrability of Hydrostatic Spheres}

\subsection{Variational Principle for Hydrostatic Spheres}

A non-rotating gaseous sphere in hydrostatic equilibrium obeys the equations of hydrostatic equilibrium and mass continuity

$$
\begin{aligned}
& -\mathrm{d} P / \rho \mathrm{d} r=G m / r^{2}, \\
& \mathrm{~d} m / \mathrm{d} r=4 \pi r^{2} \rho,
\end{aligned}
$$

where the pressure, mass density, and included mass $P(r), \quad \rho(r), m(r)$ depend on radius $r$. For dependent variables, we use the gravitational potential

$V(r)=\int_{\infty}^{r} G m / r^{2} \mathrm{~d} r$ and the thermodynamic potential (specific enthalpy, ejection energy) $H(r)=\int_{0}^{P(r)} \mathrm{d} P / \rho$, so that (5) and its integrated form become

$$
\begin{aligned}
& -\mathrm{d} H / \mathrm{d} r=\mathrm{d} V / \mathrm{d} r, \\
& V(r)+H(r)=-\frac{G M}{R},
\end{aligned}
$$

expressing the conservation of the specific energy as the sum of gravitational and internal energies, in a star of mass $M$ and radius $R$. The two first-order Equations (5) are equivalent to a second-order equation of hydrostatic equilibrium, Poisson's Law in terms of the enthalpy $H(r)$ :

$$
\frac{1}{r^{2}} \frac{\mathrm{d}}{\mathrm{d} r}\left(r^{2} \frac{\mathrm{d} H}{\mathrm{~d} r}\right)+4 \pi G \rho(H)=0,
$$

We assume a chemically homogeneous spherical structure, and thermal equilibrium in each mass shell, so that $\rho(r), P(r), H(r)$ are even functions of the radius $r$. At the origin, spherical symmetry requires $\mathrm{d} P / \mathrm{d} r=0$ and mass continuity requires, to order $r^{2}$,

$$
\begin{aligned}
& \rho(r)=\rho_{c}\left(1-A r^{2}\right), \\
& m(r)=\frac{4 \pi r^{3}}{3} \cdot\left(1-\frac{3}{5} A r^{2}\right)=\frac{4 \pi r^{3}}{3} \cdot \rho_{c}^{2 / 5} \rho(r)^{3 / 5} .
\end{aligned}
$$

The average mass density inside radius $r$ is

$$
\bar{\rho}(r):=m(r) /\left(4 \pi r^{3} / 3\right)=\rho_{c}^{2 / 5} \rho(r)^{3 / 5} .
$$

In a previous paper [2], we showed that hydrostatic equilibrium (7) follows from the variational principle $\delta W=0$ minimizing the Gibbs free energy, the integral of the Lagrangian $\mathcal{L}$ :

$$
\begin{gathered}
W:=\int_{0}^{R} \mathrm{~d} r \mathcal{L}\left(r, H, H^{\prime}\right), \\
\mathcal{L}\left(r, H, H^{\prime}\right)=4 \pi r^{2}\left[-H^{\prime 2} / 8 \pi G+P(\rho)\right] \mathrm{d} r, \\
':=\mathrm{d} / \mathrm{d} r,
\end{gathered}
$$

$W$ is the sum of the gravitational and internal specific energies per radial shell $\mathrm{d} r$. The canonical momentum and Hamiltonian,

$$
\begin{aligned}
& m:=\partial \mathcal{L} / \partial H^{\prime}=-r^{2} H^{\prime} / G, \\
& \mathcal{H}(r, H, m)=-G m^{2} / 2 r^{2}-4 \pi r^{2} P(H),
\end{aligned}
$$

are the included mass and energy per mass shell. The 
canonical equations are

$$
\begin{aligned}
& \partial \mathcal{H} / \partial m=H^{\prime}=-G m / r^{2}, \\
& \partial \mathcal{H} / \partial H=-m^{\prime}=-4 \pi r^{2} \rho .
\end{aligned}
$$

Spherical geometry makes the system nonautonomous, so that $\partial \mathcal{H} / \partial r=-\partial \mathcal{L} / \partial r=-2 \mathcal{L} / r$ vanishes only asymptotically, as the mass shells approach planarity.

The equations of hydrostatic equilibrium (5) can be rewritten

$$
\begin{aligned}
& \mathrm{d} \log u / \mathrm{d} \log r=3-u(r)-n(r) v(r), \\
& \mathrm{d} \log v / \mathrm{d} \log r=u-1+v(r)-\mathrm{d} \log [1+n(r)] / \mathrm{d} \log r,
\end{aligned}
$$

in terms of the logarithmic derivatives

$$
\begin{aligned}
& u(r):=\mathrm{d} \log m / \mathrm{d} \log r, \\
& v(r):=-\mathrm{d} \log (P / \rho) / \mathrm{d} \log r, \\
& w(r):=n(r) v(r)=-\mathrm{d} \log \rho / \mathrm{d} \log r,
\end{aligned}
$$

and an index $n(r)$

$$
\begin{aligned}
& n(r):=\mathrm{d} \log \rho / \mathrm{d} \log (P / \rho), \\
& 1+\frac{1}{n(r)}:=\mathrm{d} \log P / \mathrm{d} \log \rho,
\end{aligned}
$$

which depends on the local thermal structure. The mass density invariant $w$ makes explicit the universal mass density structure of all stellar cores, which is not apparent in the conventional pressure invariant $v$.

\subsection{Scaling Symmetry and Reduction to First-Order Equation between Scale Invariants}

Following the our results [2], a hydrostatic structure is completely integrable, if the structural Equations (5) are invariant under the infinitesimal scaling transformation

$$
\begin{aligned}
& \delta r=r, \delta \rho=-n \tilde{\omega}_{n} \rho, \delta H=-\tilde{\omega}_{n} H, \\
& \delta H^{\prime}=-\left(1+\tilde{\omega}_{n}\right) H^{\prime}, \\
& \text { where } \tilde{\omega}_{n}:=2 /(n-1),
\end{aligned}
$$

generated by the Noether charge, for constant $n$,

$$
\begin{aligned}
G_{n} & :=-\mathcal{H} \cdot r-m \cdot\left(\tilde{\omega}_{n} H\right) \\
& =r^{2}\left[\left(\frac{H^{\prime 2}}{2 G}+4 \pi P(H)\right) \cdot r+\tilde{\omega}_{n} \frac{H H^{\prime}}{G}\right] .
\end{aligned}
$$

The Lagrangian (10) then transforms as a scalar density of weight $-2 \tilde{\omega}_{n}$

$$
\delta \mathcal{L}=-2 \tilde{\omega}_{n} \mathcal{L}, \quad \delta S_{12}=\left(1-2 \tilde{\omega}_{n}\right) \cdot S_{12},
$$

so that only for the $n=5$ polytrope $\left(\tilde{\omega}_{n}=1 / 2\right)$ is the action invariant and scaling a symmetry of the action.

Both structural Equations (13) are autonomous, if and only if $n$ is constant, so that, $P(r)=K \rho(r)^{1+1 / n}$, with the same constant $K$ (related to the entropy) at each radius. When this is so ${ }^{1}$,

$$
\begin{gathered}
\mathrm{d} u / \mathrm{d} \log r=u\left(3-u-w_{n}\right), \\
\mathrm{d} w_{n} / \mathrm{d} \log r=w_{n}\left(u-1+w_{n} / n\right) \\
\frac{\mathrm{d} \log w_{n}}{u-1+w_{n} / n}=\frac{\mathrm{d} \log u}{\left(3-u-w_{n}\right)}=\mathrm{d} \log r=\frac{\mathrm{d} \log m}{u} .
\end{gathered}
$$
(20)

In this section, we consider only the first equality in

$$
\frac{\mathrm{d} w_{n}}{\mathrm{~d} u}=\frac{w_{n}\left(u-1+w_{n} / n\right)}{u\left(3-u-w_{n}\right)}
$$

between scale invariants, which encapsulates all the effects of scale invariance. We consider only simple polytropes with finite central density $\rho_{c}$, so that the regularity condition (8) requires that all $w_{n}(u)$ be tangent to $\frac{5}{3}(3-u)$ at the origin. Such Emden polytropes are the regular solutions $w_{n}(u)$ of the first-order Equation (19), for which $w_{n}(u) \rightarrow \frac{5}{3}(3-u)$ for $u \rightarrow 3$.

In terms of the dimensional constant, dimensional radius, and the central enthalpy and pressure

$$
\begin{aligned}
\alpha^{2} & :=\frac{(n+1)}{4 \pi G} K \rho_{c}^{1 / n-1}, \quad r:=\alpha \xi, \\
H_{c} & :=(n+1)(P / \rho)_{c} \equiv(n+1) K \rho_{c}^{1 / n}, \quad P_{c},
\end{aligned}
$$

the second-order equation of hydrostatic equilibrium (7), takes the dimensionless form of the Lane-Emden equation

$$
\frac{\mathrm{d}}{\mathrm{d} \xi}\left(\xi^{2} \frac{\mathrm{d} \theta_{n}}{\mathrm{~d} \xi}\right)+\xi^{2} \theta_{n}^{n}=0
$$

In terms of the dimensionless enthalpy $\theta_{n}(\xi)=H / H_{c}$, the dimensional included mass, mass density, average included mass density, and specific gravitational force are

$$
\begin{aligned}
& m(r)=4 \pi \rho_{c} \alpha^{3} \cdot\left(-\xi^{2} \theta_{n}^{\prime}\right), \\
& \rho_{n}(r)=\rho_{c} \cdot \theta_{n}^{n}(\xi), \\
& \bar{\rho}_{n}(r):=\frac{m(r)}{4 \pi r^{3} / 3}=\rho_{c} \cdot\left(-3 \theta_{n}^{\prime} / \xi\right), \\
& g(r):=4 \pi \rho_{c} \alpha^{2}\left(-\theta_{n}^{\prime}\right)
\end{aligned}
$$

where prime designates the derivative ${ }^{\prime}:=\mathrm{d} / \mathrm{d} \xi$. The

\footnotetext{
${ }^{1}$ These characteristic equations are equivalent to a predator/prey equation in population dynamics [12,13]. With time $t$ replacing-log $r$, they are Lotka-Volterra equations, modified by additional spontaneous growth terms $-u^{2}, w_{n}^{2} / n$ on the right side. The $u w$ cross-terms lead to growth of the predator $w$ at the expense of the prey $u$, so that a population that is exclusively prey initially $(u=3, w=0)$ is ultimately devoured $u \rightarrow 0$. For the weakest predator/prey interaction $(n=5)$, the predator takes an infinite time to reach the finite value $w_{5} \rightarrow 5$. For stronger predator/prey interaction $(n<5)$, the predator grows infinitely $w_{n} \rightarrow \infty$ in finite time.
} 
scale invariants are

$$
\begin{aligned}
& u:=-\xi \theta_{n}^{n} / \theta_{n}^{\prime}, \quad v_{n}:=-\xi \theta_{n}^{\prime} / \theta_{n}, \\
& \omega_{n}:=\left(u v_{n}^{n}\right)^{1 /(n-1)} \equiv-\xi^{1+\tilde{\omega}_{n}} \theta_{n}^{\prime} .
\end{aligned}
$$

The Noether charge

$$
G_{n}(\xi)=\frac{H_{c}^{2}}{G} \cdot\left\{\xi^{2} \cdot\left[\xi\left(\frac{\theta_{n}^{\prime 2}}{2}+\frac{\theta_{n}^{n+1}}{n+1}\right)+\tilde{\omega}_{n} \theta_{n} \theta_{n}^{\prime}\right]\right\},
$$

evolves radially according to

$$
\frac{\mathrm{d} G_{n}}{\mathrm{~d} \xi}=\left(1-2 \tilde{\omega}_{n}\right) \mathcal{L}=\left(\frac{H_{c}^{2}}{G}\right) \cdot\left(\frac{n-5}{n-1}\right) \cdot \xi^{2}\left(\frac{\theta_{n}^{\prime 2}}{2}-\frac{\theta_{n}^{n+1}}{n+1}\right) \cdot
$$

This non-conservation law expresses the radial evolution of energy density per mass shell, from entirely internal $\left(\theta_{n}^{n+1} /(n+1)\right)$ at the center, to entirely gravitational $\left(\theta_{n}^{\prime 2} / 2\right)$ at the stellar surface.

Figure 1 shows the first integrals $w_{n}(u)$ for $n=0,1$, $2,3,4,5$. For $n=5$, scaling is a variational symmetry so that (26) reduces to a conservation law for the Noether charge

$$
\begin{aligned}
G_{5} & =\frac{H_{c}^{2}}{G} \cdot \xi^{2}\left[\xi\left(\frac{\theta_{5}^{\prime 2}}{2}+\frac{\theta_{5}^{6}}{6}\right)+\frac{1}{2} \theta_{5} \theta_{5}^{\prime}\right] \\
& =-\frac{H_{c}^{2}}{G} \cdot\left(u v_{5}^{3}\right)^{1 / 2} \cdot\left[-v_{5}-u / 3+1\right]=\text { constant. }
\end{aligned}
$$

For the Emden solution, $v_{5}$ is finite at the stellar boundary $u=0$, the constant vanishes, and

$w_{5}(u)=5 v_{5}=\frac{5}{3}(3-u)$ everywhere.

For $n<5, v_{n}$ diverges at the stellar radius $\xi_{1}$, but $\omega_{n} \rightarrow{ }_{0} \omega_{n}$, a finite constant characterizing each Emden function. At the boundary $u=0$, our density invariant $w_{n}(u)$ diverges as $n\left[{ }_{0} \omega_{n}^{n-1} / u\right]^{1 / n}$, and

$$
\left(-\xi^{2} \theta_{n}^{\prime}\right)_{1}={ }_{0} \omega_{n} \cdot \xi_{1}^{\frac{n-3}{n-1}} .
$$

Table 1 lists these constants ${ }_{0} \omega_{n}$, along with the global mass density ratios $\rho_{c} / \bar{\rho}_{n}(R)$ and the ensuing dimensional radius-mass relation $M^{1-n}=[(n+1) K / G]^{n} \cdot\left({ }_{0} \omega_{n}^{n-1} / 4 \pi\right) R^{3-n}$. Together with the well-known $[3,5,6]$ third, fourth and fifth columns, all of this table follows directly from the regular solutions of the first-order Equation (21). In addition, the sixth and seventh columns express mass concentration in an originnal way.

\section{Increasing Polytropic Index and Mass Concentration}

Emden functions are the normalized regular solutions of the Lane-Emden Equation (23) for which the mass density is finite at the origin, so that $\theta_{n}(0)=1, \theta_{n}^{\prime}(0)=0$. Each Emden function of index $n$ is characterized by its first zero $\theta_{n}\left(\xi_{1 n}\right)=0$, at dimensionless boundary radius $\xi_{1 n}$. As an alternative measure of core concentration, we define the core radius $\xi_{\text {core }}$ implicitly by $u\left(\xi_{\text {core }}\right):=2$, where gravitational and pressure gradient forces are maximal. This core radius, where $w_{n} \approx 2$ and the mass density has fallen to $\rho_{n c o r e} / \rho_{n c} \approx 0.4$ for all polytropes $n \geq 1$, is marked by red dots in Figures 1-3. The sixth and seventh columns in Table 1 list dimensionless values for the fractional core radius $r_{n c o r e} / R=\xi_{\text {ncore }} / \xi_{1}$ and fractional included mass $m_{n \text { core }} / M$. Within the core $u>2$, the internal energy dominates over the gravitational energy, so that for $n \geq 1$,

$$
\begin{aligned}
& w_{n}(u) \approx w_{5}(u)=\frac{5}{3}(3-u), \\
& \theta_{n}(\xi) \approx 1-\xi^{2} / 6, \text { for } u_{n}>2, \xi<\xi_{\text {core }},
\end{aligned}
$$

consistent with the universal density structure (8) all stars enjoy near their center.

For $\boldsymbol{n}=\mathbf{0}$, the mass is uniformly distributed, and the entire star is core.

As $0<\boldsymbol{n}<\mathbf{5}$ increases, the radial distribution concentrates, and the envelope outside the core grows. With

Table 1. Scaling exponents, core parameters, surface parameters, and mass-radius relations for polytropes of increasing mass concentration. Columns 3 - 5 are well-known $[3,5,6]$. Columns 6 - 7 present a new measure of core concentration.

\begin{tabular}{cccccccc}
\hline$n$ & $\tilde{\omega}_{n}$ & $\xi_{1 n}$ & $\rho_{c n}(R) / \bar{\rho}_{n}(R)$ & ${ }_{0} \omega_{n}$ & $r_{n \text { corc }} / R=\xi_{\text {ncorc }} / \xi_{1}$ & $m_{n \text { corc }} / M$ & $\begin{array}{c}\text { Radius-Mass Relation } \\
R^{3-n} \sim M^{1-n} / \omega_{n}\end{array}$ \\
\hline 0 & -2 & 2.449 & 1 & 0.333 & 1 & 1 & $R \sim M^{1 / 3} ;$ mass uniformly distributed \\
1 & $\pm \infty$ & 3.142 & 3.290 & $\ldots$ & 0.66 & 0.60 & $R$ independent of $M$ \\
1.5 & 4 & 3.654 & 5.991 & 132.4 & 0.55 & 0.51 & $M^{-1 / 3}$ \\
2 & 2 & 4.353 & 11.403 & 10.50 & 0.41 & 0.41 & $M$ independent of $R$ \\
3 & 1 & 6.897 & 54.183 & 2.018 & 0.24 & 0.31 & 0.24 \\
4 & $2 / 3$ & 14.972 & 622.408 & 0.729 & 0.13 & 0.22 & 0.19 \\
4.5 & $4 / 7$ & 31.836 & 6189.47 & 0.394 & 0.08 & $R=\infty$ for any $M$; mass infinitely \\
concentrated
\end{tabular}




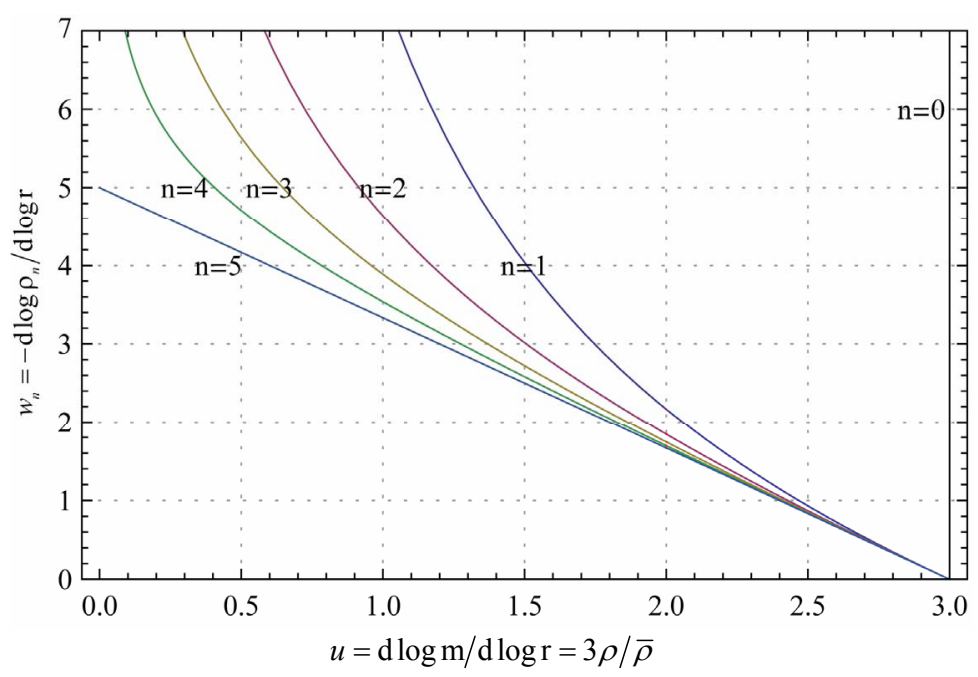

Figure 1. Dilution of polytrope mass density as the boundary is approached $(u \rightarrow 0)$. All solutions are tangent to the same density structure $w_{n}(z) \rightarrow w_{5}=(5 / 3)(3-u)$ at the center $(u=3)$, but differ for $u<2$ outside the core. Approaching the outer boundary $\left(u_{n} \rightarrow 0\right)$, the density $\rho_{n}(r)$ falls rapidly, but $u v_{n}^{n}:=\omega_{n}^{n-1}$ approaches a constant ${ }_{0} \omega_{n}^{n-1}$ so that $w_{n} \rightarrow n\left[{ }_{0} \omega_{n}^{n-1} / u_{n}\right]^{1 / n}$ diverges, for $n<5$.

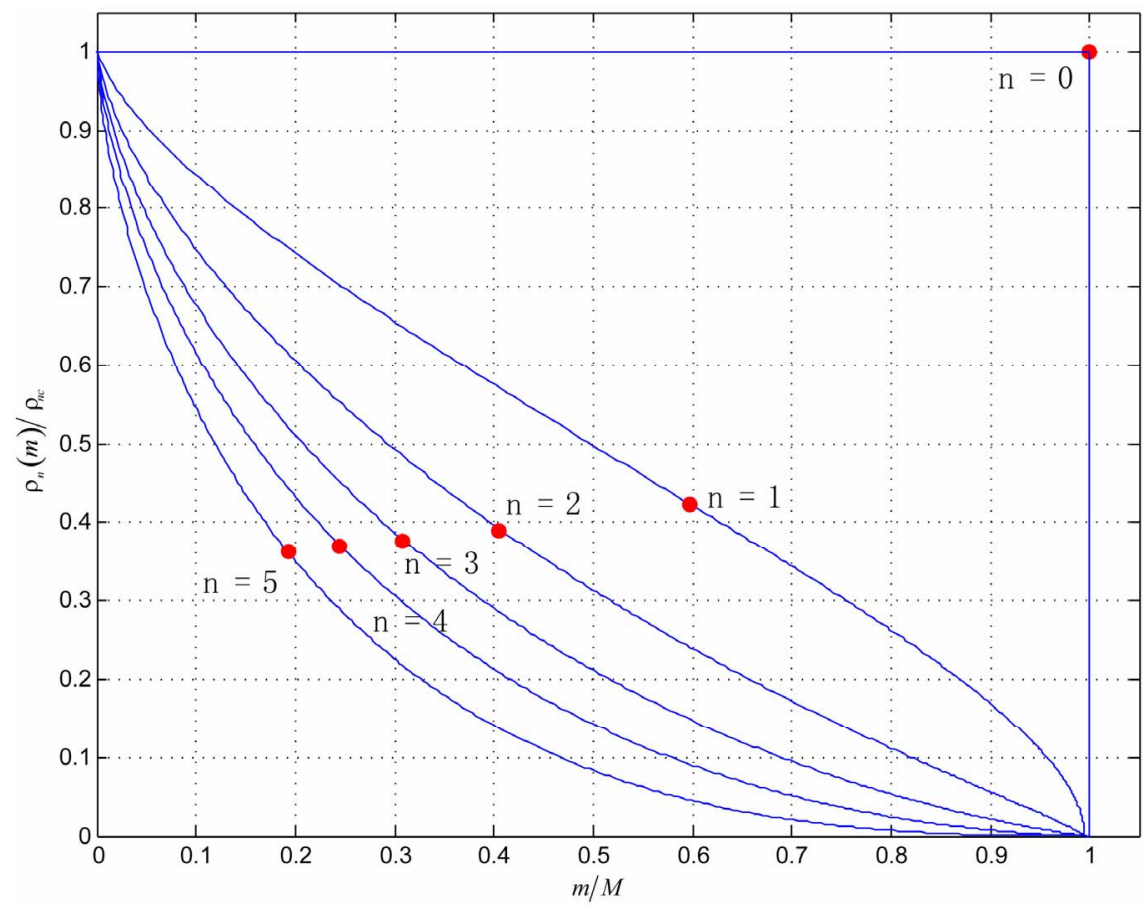

Figure 2. Normalized mass density profiles as a function of fractional included mass $m / M$, for polytropes of mass concentration increasing with $n$. The red dots mark the core radii, at which the densities stay near $\rho\left(r_{\text {core }}\right) / \rho_{c} \approx 0.4$, for all $n \geq 1$. For uniformly distributed mass $(n=0)$, the polytrope is all core. As the mass concentration increases $(n \rightarrow 5)$, the core shrinks to about $20 \%$ of the mass.

increasing core concentration:

For $1<\boldsymbol{n}<\mathbf{3}$, the radius $R$ decreases with mass $M$. Nonrelativistic degenerate stars have $n=3 / 2$.

For $\boldsymbol{n}=\mathbf{3}$, the radius $R$ is independent of mass $M$. This astrophysically important case is discussed in Section 4 and the Appendix.

For $\boldsymbol{n}>\mathbf{3}$, the radius $R$ increases with mass $M$. As $n \rightarrow 5$, the stellar radius increases $\xi_{1 n} \rightarrow 3(n+1) /(5-n)$, 


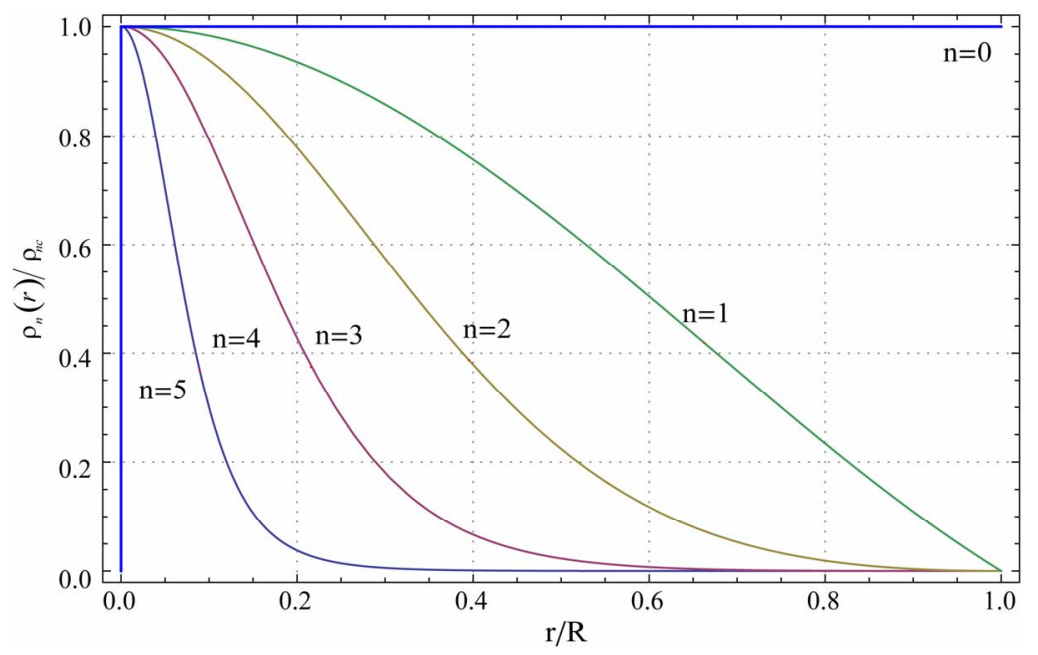

Figure 3. Normalized mass density profiles as function of fractional radius $r / R$. The density is uniform for $n=0$, but is maximally concentrated at finite radius for the $n=5$ polytrope, which is unbounded $(R=\infty)$. The density at the core radius stays about $\rho\left(r_{\text {core }}\right) / \rho_{c} \sim 0.4$, for any $n \geq 1$.

the core radius shrinks $\xi_{\text {core }} \rightarrow \sqrt{10 / 3 n}$, the fractional core radius $r_{\text {core }} / R=\xi_{\text {core }} / \xi_{1 n} \rightarrow 0.045(5-n)$, $m_{n \text { core }} / M \rightarrow 0.19$, and ${ }_{0} \omega_{n} \rightarrow \sqrt{3 / \xi_{1 n}} \rightarrow 0$.

For $\boldsymbol{n}=\mathbf{5}$, the mass is infinitely concentrated toward the center, and the stellar radius $R=\infty$ for any mass $M$. Scaling becomes a variational symmetry, so that the Noether charge $G_{5}$ in (40) is constant with radius. For the regular solution this constant vanishes:

$$
\begin{aligned}
\xi_{\text {core }} & \rightarrow \sqrt{10 / 3 n} G_{5} \sim\left[\xi\left(\frac{\theta_{5}^{\prime 2}}{2}+\frac{\theta_{5}^{6}}{6}\right)+\frac{1}{2} \theta_{5} \theta_{5}^{\prime}\right] \\
& =\left(u v_{5}^{3}\right)^{1 / 2} \cdot\left(v_{5}-u / 3-1\right)=0,
\end{aligned}
$$

so that $v_{5}=1-u / 3, \theta_{5}^{\prime}=-\xi \theta_{5}^{3} / 3$. Integrating then yields

$$
\theta_{5}(\xi)=\left(1+\xi^{2} / 3\right)^{-1 / 2}
$$

after normalizing to $\theta_{5}(0)=1$.

For $\boldsymbol{n}>\mathbf{5}$, the central density diverges, so that the total mass $M$ is infinite.

\section{Regular Emden Solutions and Their Approximations}

In place of $u$, we now introduce an equivalent homology invariant $z:=3-u=-\mathrm{d} \log \bar{\rho}_{n} / \mathrm{d} \log r$, where $\bar{\rho}_{n}:=3 m(r) / 4 \pi r^{3}$ is the average mass density inside radius $r$. In term of $z, w_{n}$, the characteristic differential Equations (20) are

$$
\frac{\mathrm{d} z}{(3-z)\left(w_{n}-z\right)}=\frac{\mathrm{d} \log w_{n}}{2-z+w_{n} / n}=\mathrm{d} \log r=\frac{\mathrm{d} \log m}{3-z} .
$$

Incorporating the boundary condition, the first of Equations (40) takes the form of a Volterra integral equa- tion [9]

$$
\begin{aligned}
w_{n}(z) & =\int_{0}^{z} \mathrm{~d} z w_{n} \frac{\left(2-z+w_{n} / n\right)}{(3-z)\left(w_{n}-z\right)} \\
& \approx\left(5 / J_{n}\right)\left[1-(1-z / 3)^{J_{n}}\right]:=w_{n \mathrm{Pic}}(z), \\
J_{n}:= & (9 n-10) /(7-n) .
\end{aligned}
$$

The Picard approximation is defined by inserting the core values $w_{n}(z) \approx(5 / 3) z$ inside the preceding integral. For $n=0,5$, this Picard approximation is everywhere exact. For intermediate polytropic indices $0<n<$ 5 , the Picard approximation breaks down approaching the boundary, where $w_{n}$ diverges as $w_{n} \rightarrow n\left[{ }_{0} \omega_{n}^{n-1} / u\right]^{1 / n}$, and is poorest for $n \approx 3$.

After obtaining $w_{n}(z):=-\mathrm{d} \log \rho_{n} / \mathrm{d} \log r$, either numerically or by Picard approximation, another integration gives [9]

$$
\begin{aligned}
& \rho_{n}(z) / \rho_{c n}=\exp \left\{-\int_{0}^{z} \frac{\mathrm{d} z w_{n}(z)}{\left[w_{n}(z)-z\right](3-z)}\right\} \\
& \approx(1-z / 3)^{5 / 2} \\
& \theta_{n}= {\left[\rho_{n}(z) / \rho_{c n}\right]^{1 / n} } \\
&= \exp \left\{-\int_{0}^{z} \frac{\mathrm{d} z w_{n}(z)}{n\left[w_{n}(z)-z\right](3-z)}\right\} \\
& \approx(1-z / 3)^{5 / 2 n}:=\theta_{n \mathrm{Pic}} \\
& m(z) / M=\left(\frac{z}{3}\right)^{3 / 2} \cdot \exp \left\{\int_{3}^{z} \mathrm{~d} z\left\{\frac{1}{\left[w_{n}(z)-z\right]}-\frac{3}{2 z}\right\}\right\} \\
& \approx\left(\frac{z}{3}\right)^{3 / 2}
\end{aligned}
$$




$$
\begin{aligned}
& r(z) / R=\xi / \xi_{1 n} \\
& =\left(\frac{z}{3}\right)^{1 / 2} \cdot \exp \left\{\int_{3}^{z} \mathrm{~d} z\left\{\frac{1}{(3-z)\left[w_{n}(z)-z\right]}-\frac{1}{2 z}\right\}\right\} \\
& \approx \frac{(3 z)^{1 / 2}}{3-z} .
\end{aligned}
$$

All the scale dependance now appears in the integration constants $M$ and $R(M)$, which except for $n=3$ depends on $M$. Inserting the core values $w_{n}(z) \approx(5 / 3) z$ inside the integral, the Picard approximations

$$
\theta_{n \mathrm{Pic}}(\xi)=\left(1+\xi^{2} / 6 N_{n}\right)^{-N_{n}}, N_{n}:=5 /(3 n-5)
$$

to the Emden functions are obtained and tabulated in the last column of Table 2. For polytropic indices $n=0,5$, this Picard form is exact. For intermediate polytropic indices $0<n<5$, the Picard approximation remains a good approximation through order $\xi^{6}$, but breaks down approaching the outer boundary. Unfortunately, the Picard approximation is poorest near $n=3$, the astrophysically most important polytrope. Figure 4 compares three approximations to this most important Emden function, shown in yellow, whose Taylor series expansion is

$$
\begin{aligned}
\theta_{3}(\xi)= & 1-\xi^{2} / 6+\xi^{4} / 40-(19 / 5040) \xi^{6} \\
& +(619 / 1088640) \xi^{8} \\
& -(2743 / 39916800) \xi^{10}+\cdots
\end{aligned}
$$

to this Taylor series expansion

$$
\begin{aligned}
& 1-0.1666667 \xi^{2}+0.025 \xi^{4}-0.0037698 \xi^{6} \\
& +0.0005686 \xi^{8}-0.00006872 \xi^{10},
\end{aligned}
$$

shown in red, diverges badly for $\xi>2.5 \approx 1.7 \xi_{3 \text { core }}$.

$$
\begin{aligned}
& \theta_{3 \text { Pic }}(\xi)=\left(1+2 \xi^{2} / 15\right)^{-5 / 4} \\
& =1-\xi^{2} / 6+\xi^{4} / 40-13 \xi^{6} / 3600+\cdots \\
& =1-0.1666667 \xi^{2}+0.025 \xi^{4}-0.003611 \xi^{6}+\cdots,
\end{aligned}
$$

shown in dashed green, converges and remains a good approximation over the bulk of the star, with $\leq 10 \%$ error out to $\xi \approx 3.9$, more than twice the core radius and more than half-way out to the stellar boundary at $\xi_{13}=6.897$.

Table 2. Taylor series and picard approximations $\theta_{n \mathrm{Pic}}(\xi)$ to emden functions $\theta_{n}(\xi)$.

\begin{tabular}{cccc}
\hline$n$ & Emden Function $\theta_{n}(\xi)$ and Taylor Series & $N_{n}:=5 /(3 n-5)$ & Picard Approximation $\theta_{n \mathrm{ric}}(\xi):=\left(1+\xi^{2} / 6 N_{n}\right)^{-N_{n}}$ \\
\hline 0 & $1-\xi^{2} / 6$ & -1 & $1-\xi^{2} / 6$ \\
1 & $\sin \xi / \xi=1-\xi^{2} / 6+\xi^{4} / 120-\xi^{6} / 5040+\cdots$ & $-5 / 2$ & $\left(1-\xi^{2} / 15\right)^{5 / 2}=1-\xi^{2} / 6+\xi^{4} / 120-\xi^{6} / 10800+\cdots$ \\
$n$ & $1-\xi^{2} / 6+n \xi^{4} / 120-n(8 n-5) / 15120 \xi^{6}+\cdots$ & $5 /(3 n-5)$ & $\left(1+\xi^{2} / 6 N_{n}\right)^{-N_{n}}=1-\xi^{2} / 6+n \xi^{4} / 120-n(6 n-5) \xi^{6} / 10800+\cdots$ \\
5 & $\left(1+\xi^{2} / 3\right)^{-1 / 2}$ & $1 / 2$ & $\left(1+\xi^{2} / 3\right)^{-1 / 2}$ \\
\hline
\end{tabular}

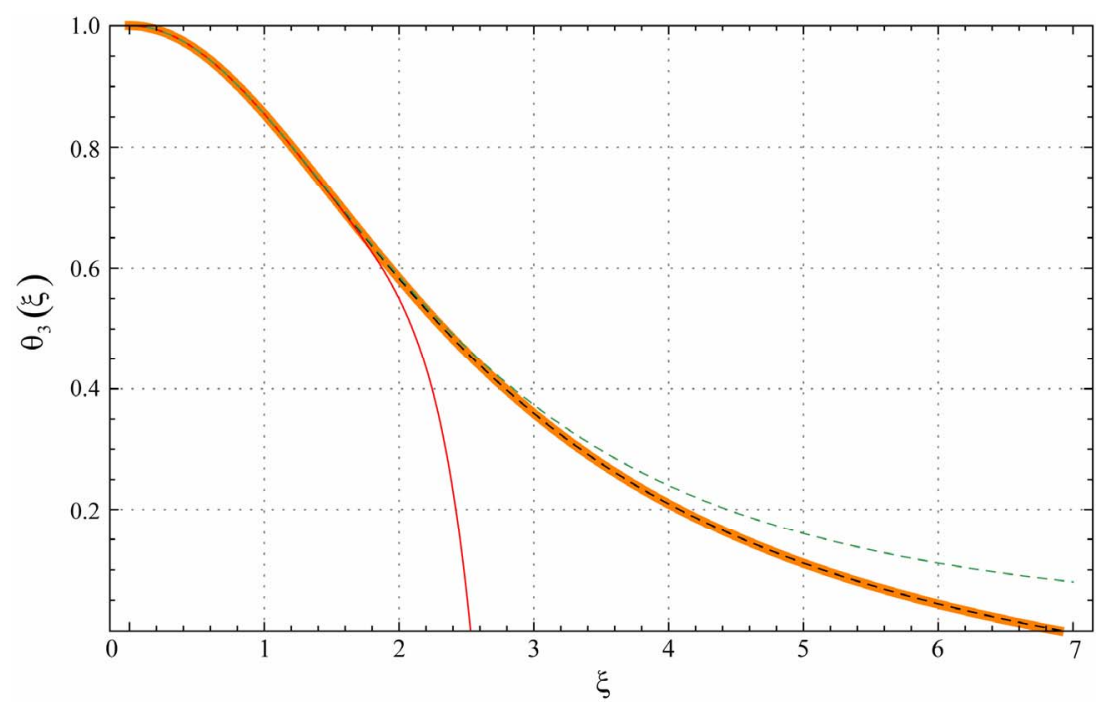

Figure 5. The exact Emden function $\theta_{3}(\xi)$ (solid yellow) and its polynomial (red), Picard (green dashed) and Padé (heavy black dashed) approximations. Even in this worst case, the Picard approximation holds out to twice the core radius at $2 \xi_{3 c o r e}=$ 3.3, before breaking down near the boundary. The Padé approximation is indistinguishable from the exact solution, vanishing at $\xi_{1}=6.921$, very close to the true boundary at $\xi_{13}=6.897$. 
This approximation suffices in white dwarf and ZAMS stars, except for their outer envelopes, which are never polytropic and contain little mass. Because it satisfies the central boundary condition, but not the outer boundary condition, the Picard approximation underestimates $\theta^{\prime}(\xi)$ and overestimates $\theta(\xi)$ outside $\xi \sim 3.9$.

Padé rational approximation $[10,11]$ :

$$
\begin{aligned}
\theta_{3 \mathrm{Pad}}= & \frac{1-\xi^{2} / 108+11 \xi^{4} / 45360}{1+17 \xi^{2} / 108+\xi^{4} / 1008} \\
= & 1-0.166667 \xi^{2}+0.025 \xi^{4}-0.00376984 \xi^{6} \\
& +0.0005686 \xi^{8}-0.0000857618 \xi^{10}+\cdots,
\end{aligned}
$$

shown in dashed heavy black, is a simpler and much better approximation. By construction, it agrees with the series expansion (40) through fourth order. In fact, this Padé approximation is almost exact out to its first zero at $\xi_{1}=6.921$, very close to the true outer boundary $\xi_{13}=$ 6.897 .

These simple analytic approximations to $\theta_{3}(\xi)$ simplify structural modeling of massive white dwarfs and ZAMS stars.

\section{Conclusions}

We have explored how a symmetry of the equations of motion, but not of the action, reduces a second-order differential equation to first-order, which can be integrated by quadrature. In scale-invariant hydrostatics, the symmetry of the equations yields a first integral, which is a first-order equation between scale invariants, and yields directly all the familiar properties of polytropes.

We observe that, like all stars, polytropes of index $n$ share a common core density profile and defined a core radius outside of which their envelopes differ. The Emden functions $\theta_{n}(\xi)$, solutions of the Lane-Emden equation that are regular at the origin, are finally obtained, along with useful approximations.

The Appendix reviews the astrophysically most important $n=3$ polytrope, describing relativistic white dwarf stars and zero age main sequence stars. While reviewing these well-known applications $[5,6]$, we stress how these same mechanical structures differ thermodynamically and the usefulness of our original (Section IV) approximations to these Emden functions.

\section{Acknowledgements}

Thanks to Andrés E. Guzmán (Universidad de Chile) for calculating the figures with Mathematica and proofreading the manuscript. SAB was supported by the Millennium Center for Supernova Science through grant P06045-F funded by Programa Bicentenario de Ciencia y Tecnología de CONICYT and Programa Iniciativa Científica Milenio de MIDEPLAN.

\section{REFERENCES}

[1] G. W. Bluman and S. C. Anco, "Symmetry and Integration Methods for Differential Equations," Springer-Verlag, Berlin, 2010.

[2] S. Bludman and D. C. Kennedy, "Invariant Relationships Deriving from Classical Scaling Transformations," Journal of Mathematical Physics, Vol. 52, 2011, Article ID: 042092.

[3] S. Chandrasekhar, "An Introduction to the Study of Stellar Structure, Chapters III, IV," University of Chicago, 1939.

[4] M. Schwarzschild, "Structure and Evolution of the Stars," Princeton University Press, Princeton, 1958.

[5] R. Kippenhahn and A. Weigert, "Stellar Structure And Evolution," Springer-Verlag, Berlin, 1990.

[6] C. J. Hansen and S. D. Kawaler, "Stellar Interiors: Physical Principles, Structure, and Evolution," Springer-Verlag, Berlin, 1994.

[7] G. P. Horedt, "Polytropes: Applications in Astrophysics and Related Fields," Kluwer, Dordrecht, 2004.

[8] F. K. Liu, "Polytropic Gas Spheres: An Approximate Analytic Solution of the Lane-Emden Equation," Monthly Notices of the Royal Astronomical Society, Vol. 281, No. 4, 1996, pp. 1197-1205.

[9] S. A. Bludman and D. C. Kennedy, "Analytic Models for the Mechanical Structure of the Solar Core," The Astrophysical Journal, Vol. 525, No. 2, 1999, pp. 1024-1031.

[10] P. Pascual, "Lane-Emden Equation and Padé's Approximants," Astronomy \& Astrophysics, Vol. 60, 1977, pp. 161-163.

[11] Z. F. Seidov, "Lane-Emden Equation: Picard vs Pade," arXiv:astro-ph/0107395.

[12] W. E. Boyce and R. C. DiPrima, "Elementary Differential Equations and Boundary Value Problems," 7th Edition, John Wiley and Sons, Hoboken, 2001.

[13] D. W. Jordon and P. Smith, "Nonlinear Ordinary Differential Equations," 3rd Edition, Oxford University Press, Oxford, 1999. 


\section{Appendix: Astrophysical Applications of the $n=3$ Polytrope}

The $n=3$ polytrope, which is realized in white dwarfs of maximum mass and in the Eddington standard model for ZAMS stars just starting hydrogen burning, is distinguished by a unique $M-R$ relation: the mass $M=4 \pi\left({ }_{0} \omega_{3}\right)(K / \pi G)^{3 / 2}$ is independent of radius $R$, but depends on the constant $K:=P / \rho^{4 / 3}$. In these stars, the gravitational and internal energies cancel, making the total energy $W=\Omega+U=0$. Because these stars are in neutral mechanical equilibrium at any radius, they can expand or contract homologously.

\subsection{Relativistic Degenerate Stars: $K$ Fixed by Fundamental Constants}

The most massive white dwarfs are supported by the degeneracy pressure of relativistic electrons, with number density $n_{e}=\rho / \mu_{e} m_{H}$, where $m_{H}$ is the atomic mass unit and the number of electrons per atom $\mu_{e}=Z / A=2$, because these white dwarfs are composed of pure He or $\mathrm{C}^{12} / \mathrm{O}^{16}$ mixtures. Thus,

$K_{W D}=(h c / 8)[3 / \pi]^{1 / 3} m_{H} \mu_{e}^{-4 / 3}$ depends only on fundamental constants. This universal value of $K_{W D}$ leads to the limiting Chandrasekhar mass

$$
\begin{aligned}
M_{\mathrm{Ch}} & =\left(\pi^{2} / 8 \sqrt{15}\right) M_{\star} / \mu_{e}^{2}=5.824 M_{\odot} / \mu_{e}^{2} \\
& =1.456 M_{\odot} \cdot(2 / \mu)^{2}
\end{aligned}
$$

\subsection{Zero-Age Main Sequence Stars: Mass and $K(M)$ Dependent on Specific Radiation Entropy}

In an ideal gas supported by both gas pressure $P_{\text {gas }}=\mathcal{R} \rho T / \mu:=\beta P$ and radiation pressure $P_{\text {rad }}=a T^{4} / 3:=(1-\beta) P$, the radiation/gas pressure ratio is

$$
\frac{P_{\mathrm{rad}}}{P_{\mathrm{gas}}}:=\frac{1-\beta}{\beta}=\frac{T^{3}}{\rho} \cdot \frac{a \mu}{3 \mathcal{R}} .
$$

The specific radiation and ideal monatomic gas entropies are

$$
S_{\mathrm{rad}}=\frac{4 a T^{3}}{3 \rho}, \quad S_{\mathrm{gas}}(r)=\left(\frac{\mathcal{R}}{\mu}\right) \cdot \log \left[\frac{T(r)^{5 / 2}}{\rho(r)}\right],
$$

so that the gas entropy gradient

$$
\frac{\mathrm{d} S_{\text {gas }}}{\mathrm{d} \log P}=\left(\frac{5 \mathcal{R}}{2 \mu}\right) \cdot\left(\nabla-\nabla_{a d}\right)=\left(\frac{\mathcal{R}}{\mu}\right) \cdot\left(\frac{\nabla}{\nabla_{a d}}-1\right)
$$

depends on the difference between the adiabatic gradient $\nabla_{a d}=2 / 5$ and the star's actual thermal gradient $\nabla:=\mathrm{d} \log T / \mathrm{d} \log P$, which depends on the radiation transport.

Bound in a polytrope of order $n$, the ideal gas thermal gradient and gas entropy gradient are

$$
\nabla:=1 /(n+1), \quad \frac{\mathrm{d} S_{\mathrm{gas}}}{\mathrm{d} \log P}=\left(\frac{\mathcal{R}}{\mu}\right) \cdot\left[\frac{5}{2(n+1)}-1\right] .
$$

For $n>3 / 2$, the thermal gradient is subadiabatic, the star's entropy increases outwards, so that the star is stable against convection.

ZAMS stars, with mass $0.4 M_{\odot}<M<150 M_{\odot}$, have nearly constant radiation entropy $S_{\text {rad }}(M)$, because radiation transport leaves the luminosity generated by interior nuclear burning everywhere proportional to the local transparency (inverse opacity) $\kappa^{-1}$. Assuming constant $S_{\text {rad }}(M)$, we have Eddington's standard model, an $n=3$ polytrope with $S_{\text {rad }}(M)=4(\mathcal{R} / \mu) \cdot(1-\beta) / \beta$ and

$$
K(M)=P / \rho^{4 / 3}=\left\{[3(1-\beta) / a](\mathcal{R} / \mu \beta)^{4}\right\}^{1 / 3},
$$

depends only on $\beta(M)$, which is itself determined by Eddington's quartic equation $[3,5,6]$

$$
\begin{aligned}
& \frac{1-\beta}{\beta^{4}}=\left(\frac{M \mu^{2}}{M_{\star}}\right)^{2}, \\
& M_{\star}:=\frac{3 \sqrt{10}{ }_{0} \omega_{3}}{\pi^{3}}\left(\frac{h c}{G m_{H}^{4 / 3}}\right)^{3 / 2}=18.3 M_{\odot} .
\end{aligned}
$$

The luminosity

$$
\begin{aligned}
L & =L_{\text {Edd }}[1-\beta(M)] \\
& =L_{\text {Edd }} \cdot(0.003) \mu^{4} \beta(M)^{4}\left(M / M_{\odot}\right)^{3},
\end{aligned}
$$

depends on the Eddington luminosity $L_{\mathrm{Edd}}:=4 \pi c G M / \kappa_{p}$ through the photospheric opacity $\kappa_{p}$. This mass-luminosity relation is confirmed in ZAMS stars: on the lowermass ZAMS, $\beta \approx 1, L \sim M^{3} ;$ on the upper-mass ZAMS, $\beta \approx\left(M \mu^{2} / M_{\star}\right)^{-2} \ll 1, L \sim M \quad[6]$. 